\title{
Association of antinuclear antibody seropositivity with inhaled environmental exposures in patients with interstitial lung
}

\section{disease}

\author{
Kathleen Biblowitz ${ }^{1}$, Cathryn Lee ${ }^{2}$, Daisy $\mathrm{Zhu}^{3}$, Imre Noth ${ }^{3}$, Rekha Vij ${ }^{2}$, Mary E. Strek ${ }^{2}$,
} Shashi K. Bellam ${ }^{4}$ and Ayodeji Adegunsoye ${ }^{2}$

${ }^{1}$ Division of Pulmonary and Critical Care, Dept of Medicine, Thomas Jefferson University, Philadelphia, PA, USA. ${ }^{2}$ Section of Pulmonology and Critical Care Medicine, University of Chicago, Chicago, IL, USA. ${ }^{3}$ Pulmonary and Critical Care Medicine, University of Virginia, Charlottesville, VA, USA. ${ }^{4}$ Division of Pulmonary and Critical Care, Dept of Medicine, NorthShore University HealthSystem, Evanston, IL, USA.

Corresponding author: Kathleen Biblowitz (kmbiblowitz@gmail.com)

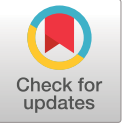

Copyright $\odot$ The authors 2021

This version is distributed under the terms of the Creative Commons Attribution NonCommercial Licence 4.0. For commercial reproduction rights and permissions contact permissions@ersnet.org

This article has supplementary material available from openres.ersjournals.com

Received: 12 April 2021 Accepted: 2 Aug 2021

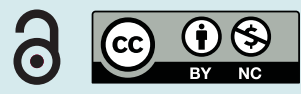

\begin{abstract}
Shareable abstract (@ERSpublications)
Association of antinuclear antibody seropositivity with inhaled exposures in ILD https://bit.ly/ 3AwIPeZ
\end{abstract}

Cite this article as: Biblowitz K, Lee C, Zhu D, et al. Association of antinuclear antibody seropositivity with inhaled environmental exposures in patients with interstitial lung disease. ERJ Open Res 2021; 7 : 00254-2021 [DOI: 10.1183/23120541.00254-2021].

\section{Abstract}

Background Interstitial lung diseases (ILDs) are diffuse parenchymal lung disorders that cause substantial morbidity and mortality. In patients with ILD, elevated antinuclear antibody (ANA) titres may be a sign of an autoimmune process. Inhalational exposures contribute to ILD pathogenesis and affect prognosis and may trigger autoimmune disease. The association of inhalational exposures with ANA seropositivity in ILD patients is unknown.

Methods This was a retrospective cohort study of adult ILD patients from five centres in the United States. Exposures to tobacco, inhaled organic antigens and inhaled inorganic particles were extracted from medical records. A multivariable logistic regression model was used to analyse the effects of confounders including age, ILD diagnosis, gender and exposure type on ANA seropositivity.

Results Among 1265 patients with ILD, there were more ANA-seropositive (58.6\%, n=741) than ANAseronegative patients $(41.4 \%, \mathrm{n}=524)$. ANA-seropositive patients had lower total lung capacity (69\% versus $75 \%, \mathrm{p}<0.001)$ and forced vital capacity $(64 \%$ versus $70 \%, \mathrm{p}<0.001)$ than patients who were ANAseronegative. Among patients with tobacco exposure, $61.4 \%(\mathrm{n}=449)$ were ANA-positive compared to $54.7 \%(n=292)$ of those without tobacco exposure. In multivariable analysis, tobacco exposure remained independently associated with increased ANA seropositivity (OR 1.38, 95\% CI 1.12-1.71). This significant difference was similarly demonstrated among patients with and without a history of inorganic exposures (OR 1.52, 95\% CI 1.12-2.07).

Conclusion Patients with ILD and inhalational exposure had significantly higher prevalence of ANAseropositivity than those without reported exposures across ILD diagnoses. Environmental and occupational exposures should be systematically reviewed in patients with ILD, particularly those with ANA-seropositivity.

\section{Background}

Interstitial lung diseases (ILDs) are diffuse parenchymal lung disorders characterised by cellular proliferation, inflammation and fibrosis or a combination of these within the alveolar wall, not due to cancer or infection [1]. ILD is associated with substantial morbidity and mortality [2].

Autoimmunity has an established connection to ILD, which is considered the primary pulmonary manifestation of multiple autoimmune diseases, and the diagnosis of ILD requires evaluation for underlying connective tissue disease (CTD) $[3,4]$. The presence of antinuclear antibodies (ANA) has been 
independently associated with ILD in previous studies, with ILD subtypes characterised by the presence of ANA serology $[5,6]$. Furthermore, inhalational exposures including smoking are known contributors to ILD and to the development of CTD [7-11].

However, there is a gap in knowledge in the link between ANA serology and inhalational exposures in ILD. In this study, we systematically assessed a cohort of ILD patients with documented ANA serology to identify prior or current exposure to inhalational antigens. We categorised the exposures into tobacco-related, organic antigens or inorganic antigens and determined whether there was an association between documented ANA serology and inhalational exposure in these patients.

Material and methods

Study population

This retrospective analysis was conducted at NorthShore University HealthSystem and University of Chicago (Chicago, IL, USA). The study was approved by the respective local institutional review board ethics committees (\#16-1062; \#17-025). Patients aged $\geqslant 18$ years from five hospital centres in the United States with a diagnosis of ILD were identified and included. ILD diagnosis was multidisciplinary and based on clinical, pulmonary, radiological and/or histopathological evaluation conducted between January 2006 and July 2016.

Data were extracted from the electronic medical records of both institutions as documented at the time of initial ILD diagnosis. Laboratory data collected included ANA titre or immunoassay screen value depending on the institution's standard. In addition, data from pulmonary function tests (PFTs) were assessed at baseline evaluation for diagnosis of ILD, including percentage predicted forced vital capacity (FVC), total lung capacity (TLC) and diffusing capacity of the lung for carbon monoxide $\left(D_{\mathrm{LCO}}\right)$.

Additional variables included demographic data (age, gender, race/ethnicity), history of exposure to tobacco (pack-years), inhaled organic antigens (such as mould, avian dander, hot tubs or paint), and inhaled inorganic particles (such as asbestos, silica or metal particles).

\section{Enrolment criteria}

All patients were from within the University of Chicago ILD registry or the NorthShore University HealthSystem and had an International Classification of Diseases (ICD)-9 or -10 billing code for ILD. Patients were included in the study if they had an available quantitative ANA titre value drawn from within the institution within 3 months of the date of diagnosis. Patients with a diagnosis of obstructive lung disease or sarcoidosis were excluded.

Patients with an ICD-9 or ICD-10 code-based ILD diagnosis at the tertiary centre (University of Chicago Hospital) underwent a multidisciplinary evaluation using available clinical data, PFTs, high-resolution computed tomography (HRCT) scans and surgical lung biopsies according to current American Thoracic Society/European Respiratory Society criteria. Pulmonologists, rheumatologists, dedicated chest radiologists and a thoracic pathologist did an assessment for the multidisciplinary diagnosis of ILD. The four nontertiary hospitals are suburban community hospitals that do not routinely perform multidisciplinary discussions for ILD diagnosis. As such, an independent "adjudication” panel of two academic pulmonologists with expertise in ILD evaluated clinical data including PFTs and HRCT scans to confirm the diagnosis of ILD in all patients who received an ICD-9 code-based ILD diagnosis from a pulmonologist at that centre [12].

\section{Autoimmune serology}

Patients with a quantitative ANA titre value were included in the study. An ANA titre value $\geqslant 1: 160$ (University of Chicago) or an immunoassay screen value $\geqslant 0.90$ (NorthShore University HealthSystem) was considered positive. One study comparing healthy patients to those with known autoimmune disease found that $5.0 \%$ of the healthy population was ANA-seropositive at 1:160 and a slightly lower 3.3\% were seropositive at 1:320 [13]. Pathology guidelines published in 2000 noted that while the use of ANA titres $>1: 160$ to define a positive test result may lead to better specificity for the diagnosis of systemic lupus erythematosus, this practice will decrease diagnostic sensitivity of the ANA test [14]. A more recent study evaluating the characteristics that distinguish autoimmune-featured (AIF)-ILD from IPF and CTD-ILD also considered ANA titres $\geqslant 1: 160$ as positive [5].

Although the sensitivity of ELISA and indirect fluorescent antibody (IFA) are similar ( $>80 \%)$, ANA IFA measured on Hep-2 cells is considered the gold standard for measuring ANA, given its much higher 
specificity (99\% versus 36-94\%) [15]. Because ELISA testing has lower specificity than IFA testing, it may have more false-positive tests.

\section{Exposure history}

The exposure history for patients at all five hospitals was systematically extracted from the electronic medical record. Data on environmental exposures for patients at the University of Chicago were obtained from the ILD registry. Data for patients at the NorthShore University HealthSystem were pulled from the electronic medical record social and occupational history. If not listed explicitly in these sections, the pulmonary physician's notes were reviewed, as were notes of other internal medicine physicians.

\section{Statistical analysis}

Continuous variables were reported as mean \pm SD and compared using a two-tailed t-test. Categorical variables were reported as counts and percentages; comparisons were made using the Chi-squared test. A p-value $<0.05$ was considered to be statistically significant. All multivariable data analyses were performed in Stata v.15 (StataCorp, College Station, TX, USA). A multivariable logistic regression model was used to analyse the effects of confounders including age, ILD diagnosis, gender and exposure type on ANA seropositivity.

As age has been variably suggested to demonstrate a linear correlation with ANA seropositivity [16, 17], subject ages were stratified into deciles to evaluate the relationship between age and ANA seropositivity. A multivariable logistic regression model was used to analyse the effects of race, age, ILD diagnosis, gender and exposure type (tobacco, organic and inorganic matter) on ANA seropositivity.

Results

Of the initial cohort of ILD patients $(n=2128)$ obtained from the University of Chicago and NorthShore Hospitals, 863 were excluded due to lack of ANA serology data, leaving 1265 patients in the study. Baseline demographics revealed the mean age at time of diagnosis was $64.7 \pm 12.3$ years. The average BMI

\section{TABLE 1 Baseline demographics of patients with interstitial lung disease (ILD)}

\begin{tabular}{|c|c|c|c|c|}
\hline & Combined & ANA-seropositive & ANA-seronegative & $p$-value ${ }^{\#}$ \\
\hline Age years & $64.7 \pm 12.3$ & $64.1 \pm 12.4$ & $65.5 \pm 12.1$ & 0.045 \\
\hline Male & $622(49.2)$ & $362(58.2)$ & $260(41.8)$ & 0.789 \\
\hline $\mathrm{BMI} \mathrm{kg} \cdot \mathrm{m}^{-2}$ & $29.8 \pm 6.6$ & $29.6 \pm 6.7$ & $30.1 \pm 6.5$ & 0.251 \\
\hline \multicolumn{5}{|l|}{ Race/ethnicity } \\
\hline Caucasian & 918 (72.6) & $515(56.1)$ & $403(43.9)$ & 0.004 \\
\hline African American & $203(16.0)$ & $147(72.4)$ & $56(27.6)$ & $<0.001$ \\
\hline Hispanic & $65(5.1)$ & $50(76.9)$ & $15(23.1)$ & 0.002 \\
\hline Asian & $47(3.7)$ & 25 (53.1) & $22(46.8)$ & 0.445 \\
\hline Other & $32(2.5)$ & $4(12.5)$ & $28(87.5)$ & $<0.001$ \\
\hline \multicolumn{5}{|l|}{ Lung function } \\
\hline TLC \% & $71.3 \pm 18.6$ & $69.0 \pm 17.2$ & $74.8 \pm 20.0$ & $<0.001$ \\
\hline FVC \% & $66.3 \pm 19.0$ & $64.1 \pm 18.4$ & $69.5 \pm 19.4$ & $<0.001$ \\
\hline$D_{\text {LCO }} \%$ & $51.0 \pm 21.7$ & $50.0 \pm 21.8$ & $52.5 \pm 21.6$ & 0.065 \\
\hline \multicolumn{5}{|l|}{ ILD subtype } \\
\hline IPF & $402(31.8)$ & $212(52.7)$ & $190(47.3)$ & 0.003 \\
\hline IPAF & $177(14.0)$ & $141(79.7)$ & $36(20.3)$ & $<0.001$ \\
\hline CTD-ILD & $240(19.0)$ & $177(73.8)$ & $63(26.3)$ & $<0.001$ \\
\hline $\mathrm{HP}$ & $141(11.2)$ & $82(58.2)$ & $59(41.8)$ & 0.914 \\
\hline Other ILD" & $96(7.5)$ & $29(30.2)$ & $67(69.8)$ & $<0.001$ \\
\hline Unclassifiable ILD & $209(16.5)$ & $100(47.8)$ & $109(52.2)$ & 0.001 \\
\hline \multicolumn{5}{|c|}{$\begin{array}{l}\text { Data are presented as mean } \pm \text { SD or } n(\%) \text {, unless otherwise stated. Exception for participants: age } n=1265 \text {, } \\
\text { gender } n=1265 \text {, antinuclear antibody (ANA) serologies } n=1265 \text {, body mass index (BMI) } n=1122 \text {, total lung } \\
\text { capacity (TLC) } n=989 \text {, forced vital capacity (FVC) } n=1162 \text {, diffusing capacity of the lung for carbon monoxide } \\
\left(D_{\text {Lco) }} n=1115 \text {, other ILD } n=95 \text {. IPF: idiopathic pulmonary fibrosis; IPAF: interstitial pneumonia with autoimmune }\right. \\
\text { features; CTD-ILD: connective tissue disease associated ILD; HP: hypersensitivity pneumonitis. \#: based on } \\
\text { univariate analysis for categorical variables, e.g. Caucasian compared to non-Caucasian, IPF compared to } \\
\text { non-IPF, etc; p-value based on two-tailed t-test for continuous variables, e.g. age, BMI; ฯ: include } \\
\text { pneumoconiosis, lymphocytic interstitial pneumonia, Langerhans cell histiocytosis, cryptogenic organising } \\
\text { pneumonia, lymphangioleiomyomatosis and other less common ILDs with small sample sizes. }\end{array}$} \\
\hline
\end{tabular}


was $29.8 \pm 6.6 \mathrm{~kg} \cdot \mathrm{m}^{-2}$, and the sex distribution of subjects was near equal (females $50.8 \%$, males $49.2 \%$ ) (table 1).

\section{Pulmonary function testing}

The majority of subjects $(77.1 \%, \mathrm{n}=975)$ underwent full PFT. The calculated mean values were TLC 71.3 $\pm 18.6 \%$, FVC $66.3 \pm 19.0 \%$ and $D_{\text {LCO }} 51.0 \pm 21.7 \%$ (table 1 ). ANA-seropositive patients had lower TLC (69\% versus $75 \%, \mathrm{p}<0.001)$ and FVC $(64 \%$ versus $70 \%, \mathrm{p}<0.001)$ than patients who were ANA-seronegative (table 1).

Autoimmune serology and inhalational exposures

Among the 1265 ILD patients in the study, there were more ANA-seropositive (58.6\%, $n=741$ ) than ANA-seronegative patients $(41.4 \%, \mathrm{n}=524)$ based on titre or immunoassay screen. An ANA-enzyme immunoassay (EIA) of 0.90 was considered an approximate equivalent to an ANA-IFA of 1:160. Of note, an ANA-EIA with a reference range of 0.9 demonstrates equivalent sensitivity and somewhat higher specificity compared to ANA-IFA (supplementary figure S1) [18].

The majority of patients reported at least one inhalational exposure (73.6\%, $n=931)$. A significant majority of patients reported a history of cigarette smoking $(57.8 \%, \mathrm{n}=731)$. Fewer subjects reported either organic exposure $(36.7 \%, n=465)$ or inorganic exposure $(16.4 \%, n=207)$.

Using a univariate analysis, an assessment of ILD patients with an exposure history revealed a higher prevalence of ANA seropositivity for all exposure subtypes (figure 1). Of the patients with tobacco exposure, $61.4 \%(n=449)$ was ANA-seropositive compared to $54.7 \%(n=292)$ of those without tobacco exposure (OR 1.32, 95\% CI 1.05-1.66). This significant difference was similarly demonstrated among patients with and without a history of organic exposure (OR 1.32, 95\% CI 1.04-1.67) and among subjects with and without inorganic exposures (OR 1.47, 95\% CI 1.08-2.01) (figure 1).

These results remained consistent upon multivariable analysis for tobacco (OR 1.38, 95\% CI 1.12-1.71), and inorganic antigen exposure (OR 1.52, 95\% CI 1.12-2.07) (table 2). However, when using the multivariable logistic regression model, organic exposure was not predictive of a positive ANA (OR 1.11, 95\% CI 0.89-1.40) (table 2).

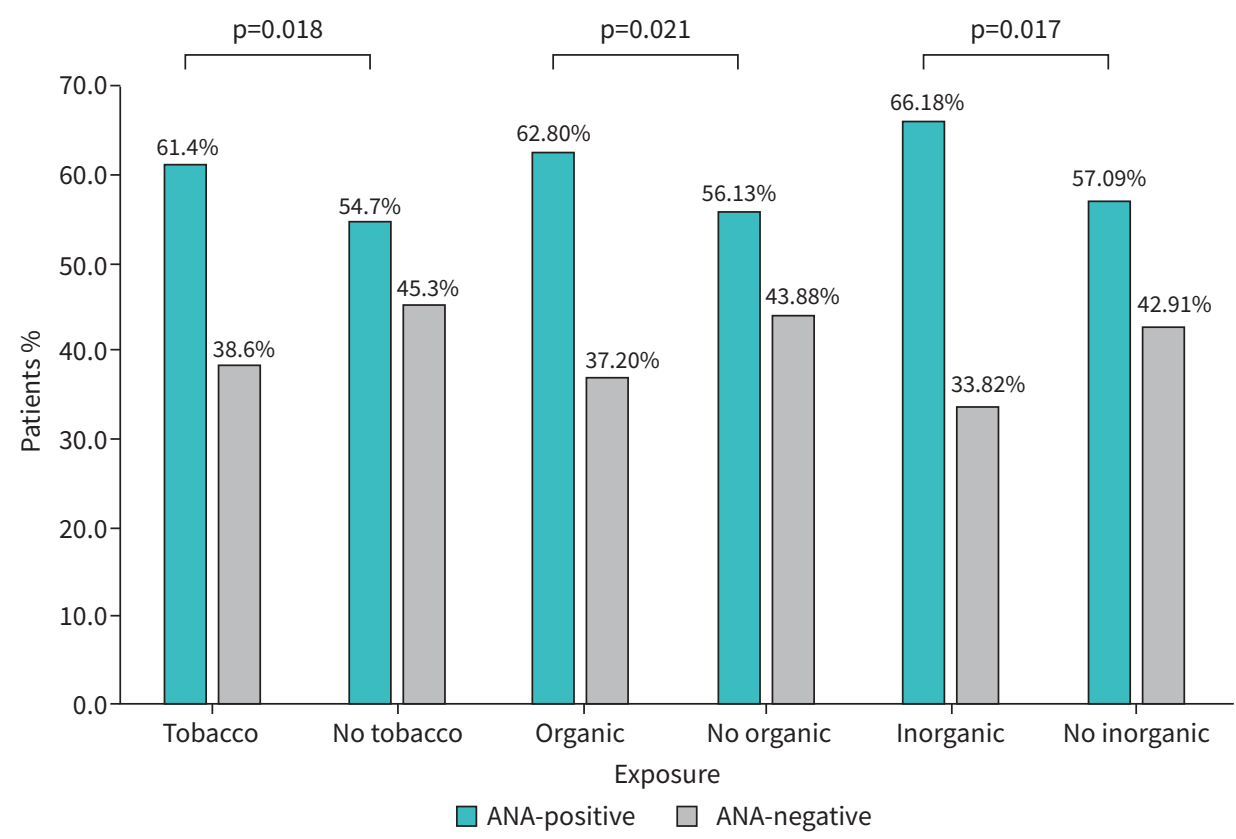

FIGURE 1 Prevalence of antinuclear antibody (ANA) seropositivity in interstitial lung disease patients with inhalational exposures. 


\begin{tabular}{|c|c|c|c|}
\hline & $\mathrm{OR}^{\#}(95 \% \mathrm{Cl})$ & Standard error & $\mathrm{p}$-value \\
\hline \multicolumn{4}{|l|}{ Diagnosis } \\
\hline IPAF & $3.56(2.48-5.13)$ & 0.22 & $<0.0001$ \\
\hline CTD-ILD & $2.56(1.82-3.60)$ & 0.21 & $<0.0001$ \\
\hline $\mathrm{HP}$ & $1.39(0.97-1.98)$ & 0.22 & 0.13 \\
\hline Other ILD & $0.44(0.29-0.67)$ & 0.25 & 0.0014 \\
\hline Unclassifiable ILD & $0.92(0.68-1.23)$ & 0.18 & 0.63 \\
\hline Age & $1.00(0.99-1.01)$ & 0.01 & 0.46 \\
\hline Male gender & $1.05(0.84-1.31)$ & 0.14 & 0.73 \\
\hline \multicolumn{4}{|l|}{ Race/ethnicity } \\
\hline African American & $1.78(1.30-2.45)$ & 0.19 & 0.003 \\
\hline Hispanic & $2.23(1.34-3.71)$ & 0.31 & 0.01 \\
\hline Asian & $1.04(0.62-1.75)$ & 0.32 & 0.89 \\
\hline Other & $0.14(0.06-0.36)$ & 0.55 & 0.0004 \\
\hline \multicolumn{4}{|l|}{ Exposure } \\
\hline Tobacco & $1.38(1.12-1.71)$ & 0.13 & 0.01 \\
\hline Organic antigen & $1.11(0.89-1.40)$ & 0.14 & 0.44 \\
\hline Inorganic antigen & $1.52(1.12-2.07)$ & 0.19 & 0.02 \\
\hline \multicolumn{4}{|c|}{$\begin{array}{l}\text { IPAF: interstitial pneumonia with autoimmune features; CTD-ILD: connective tissue disease associated } \\
\text { interstitial lung disease; HP: hypersensitivity pneumonitis. ": OR analysed using multivariable logistic regression } \\
\text { with adjustments for age, gender and race. OR comparisons were made for every increase of } 1 \text { year for age, } \\
\text { male gender in comparison to female gender, diagnosis compared to idiopathic pulmonary fibrosis, race } \\
\text { compared to Caucasians, tobacco compared to nontobacco users and exposure compared to those without } \\
\text { identified environmental exposure. }\end{array}$} \\
\hline
\end{tabular}

ANA seropositivity in hypersensitivity pneumonitis (HP) was 58.2\%, which was greater than the prevalence of positive ANA titres found in those with other ILDs (30.2\%), IPF (52.7\%) and unclassifiable ILD (47.8\%) (table 1). Of those with HP, 67.4\% ( $\mathrm{n}=95)$ had a history of organic exposure compared to those who did not have a history of organic exposures $(32.6 \%, n=46)$. This is significant when compared to patients without HP, of whom only $32.9 \%(n=370)$ had organic exposures compared to those without organic exposures $(67.1 \%, \mathrm{n}=754 ; \mathrm{p}<0.001)$ (supplementary table S3).

\section{Race/ethnicity}

Subjects were predominantly White (72.6\%) followed by Black (16.0\%), Hispanic (5.1\%) and Asian (3.7\%) (table 1). A small percentage of ILD patients (2.5\%) identified as "other". A multivariable logistic regression showed that being Black (OR 1.78, 95\% CI 1.30-2.45) or Hispanic (OR 2.23, 95\% CI 1.343.71) is highly predictive of having positive ANA. Identifying as other was associated with not having a positive ANA (OR 0.14, 95\% CI 0.058-0.36). The null hypothesis could not be rejected for Asian ethnicity (table 2).

\section{ILD diagnosis}

The prevalence of ANA seropositivity in IPF patients was $52.7 \%(n=212)$ compared to ANA-seronegative patients (47.3\%, $n=190 ; \mathrm{p}=0.003$ ). We performed a multivariable logistic regression comparatively evaluating risk of ANA seropositivity in subjects with various ILD diagnoses against a benchmark diagnosis of IPF. The regression demonstrated that the diagnosis of interstitial pneumonia with autoimmune features (IPAF) and CTD-ILD were associated with increased risk of ANA seropositivity, while a diagnosis of "other ILD" was protective. IPAF carried a higher odds ratio of 3.56 (95\% CI 2.485.13) compared to the lower odds ratio of 2.56 for CTD-ILD (95\% CI 1.81-3.60). A diagnosis of other ILD was associated with lower odds of having a positive ANA serology (OR 0.44, 95\% CI 0.29-0.67). Among those categorised as unclassifiable ILD who had combined pulmonary fibrosis and emphysema (CPFE), patients with a significant smoking history of $>10$ pack-years had numerically higher prevalence of ANA seropositivity $(n=16,50.0 \%)$ than ANA seronegativity $(n=4,23.5 \%$; $p=0.073)$, but this was not statistically significant (supplementary table S4).

\section{Pneumoconiosis}

Electronic medical record documentation regarding a secondary diagnosis of pneumoconiosis was available for review in 1045 patients of which $8.1 \%(n=85)$ had pneumoconiosis. Among these patients with a 
secondary diagnosis of pneumoconiosis, more patients had ANA seropositivity ( $n=64,9.1 \%)$ than those having ANA seronegativity ( $\mathrm{n}=21,6.2 \%$; $\mathrm{p}=0.12$ ) (supplementary table $\mathrm{S} 5$ ). Among the subset classified as having other ILD we identified six patients who also had a secondary diagnosis of pneumoconiosis. Five of these six patients had ANA seropositivity ( $n=5,83.3 \%)$. Among the remaining 30 patients with other ILD without pneumoconiosis, fewer had ANA seropositivity $(n=13,72.2 \%)$ than those with ANA seronegativity ( $\mathrm{n}=17,94.4 \% ; \mathrm{p}=0.074)$ (supplementary table S5).

\section{Discussion}

In this study, we report that ILD patients with a history of inhalational exposure had a significantly higher prevalence of ANA seropositivity than those without reported exposures. Demographics of the cohort revealed mean pulmonary function test values consistent with moderate lung dysfunction. To our knowledge, this is the first multicentre demonstration of an association between ANA serology and inhalational exposures in ILD.

African American and Hispanic race within the entire ILD cohort were both highly predictive of having a positive ANA, consistent with retrospective cohort studies showing that patients of African ancestry have the greatest prevalence of seropositive ANA titres and CTD-ILD compared to other racial groups [12, 19]. There may be a role for structural racism in this discrepancy, in that patients who are of African American and Hispanic race are more likely to live in areas where they may be exposed to ambient pollution, which could then increase the risk of autoimmunity $[20,21]$.

The majority of patients in this study, all of whom carried a diagnosis of ILD, were ANA seropositive. ANA serology was used as a marker of a systemic autoimmune response and an ANA titre of 1:160 or higher was considered seropositive [22].

ILD has an established association with autoimmunity. This is evident in ILD patients diagnosed with CTD or presenting with autoimmune features, classified as having subtypes of CTD-ILD, AIF-ILD or IPAF. In addition, ILD is the primary pulmonary manifestation of multiple autoimmune conditions including rheumatoid arthritis, scleroderma (systemic sclerosis), inflammatory myositis (polymyositis and dermatomyositis), Sjögren’s syndrome, and undifferentiated connective tissue disease [3].

The majority of patients in our cohort had at least one reported exposure. However, this is not a novel finding, because numerous ILDs have been linked to environmental exposures. HP can manifest from exposure to a variety of inhaled antigens, which results in diffuse inflammation of small airways and pulmonary parenchyma [8]. This study demonstrated that ANA seropositivity in HP was higher than those with other ILD, IPF and unclassifiable ILD. However, ILDs such as IPAF and CTD-ILD had higher ANA-seropositive prevalence because they required an ANA-seropositive value for diagnosis. Notably, this study showed that patients with HP have a higher prevalence of inhaled organic particles than patients with another ILD diagnosis. As with HP, pneumoconioses are lung diseases related to inhalational exposures, specifically mineral dusts such as silica, coal mining dust, asbestos and beryllium [8]. Our study demonstrated that ILD patients with a history of one or more inhalational exposures were more likely to be ANA-seropositive than those without a reported exposure. This was true for the univariate analysis of tobacco, organic and inorganic exposures and ANA. However, the association was not present for organic exposures in our multivariable analysis. This might suggest a weaker association between organic antigen exposures such as mould and ANA serology [12, 13].

Additionally, this study identified patients with a secondary diagnosis of pneumoconiosis and found that among those with pneumoconiosis, there was a higher prevalence of ANA seropositivity than ANA seronegativity, although this was not statistically significant. The disproportionately higher prevalence of ANA seropositivity further supports the idea that ANA titres are associated with environmental exposures in patients with ILD. In addition, we demonstrated an association between tobacco exposure and ANA seropositivity in the entire cohort. Interestingly, among those with unclassifiable ILD, patients with CPFE and a heavy smoking history had a high prevalence of ANA seropositivity compared to ANA seronegativity, further strengthening the relationship with tobacco exposure.

There are several studies that corroborate an association between the presence of autoimmune serology and inhalational exposures, but not exclusively in patients with a diagnosis of ILD. A case report of patients in Libby, MT, USA, with a history of mining asbestos-contaminated vermiculite reported a higher frequency of ANA seropositivity in miners compared to healthy patients. In addition, it demonstrated a positive correlation between ANA titres, lung disease severity and extent of exposure, suggesting a relationship between autoimmune response and asbestos-related disease processes [23]. A retrospective study of 
Brazilian silica-exposed workers found that silica may nonspecifically enhance the immune response, supporting the association between silica exposure and autoimmune disorders [24]. The diagnosis of silicosis in these workers was based on confirmation of exposure and the presence of pulmonary parenchyma alterations, but not a multidisciplinary diagnosis of ILD. Furthermore, multiple epidemiological studies including meta-analyses have identified cigarette smoking as an important risk factor for rheumatoid arthritis [25, 26]. Rheumatoid arthritis is serologically characterised by the presence of autoantibodies against factors such as rheumatoid factor and cyclic citrullinated peptide. Cigarette smoking is a known trigger of immune reactions to autoantigen modified by citrullination [27]. Additionally, animal model studies have shown administration of various heavy metals induce production of autoantibodies and polyclonal activation of the immune system in genetically susceptible rats [28].

The association between autoimmunity and exposure in ILD patients raises questions about the pathophysiology of ILD. Specifically, it remains unknown whether the presence of autoantibodies in ILD patients represents a heightened immune response and is a protective influence or if it represents a dysregulated immune response. Interestingly, the presence of autoantibodies in patients with ILD of known aetiology, including inhalational exposure (tobacco, organic, and inorganic), CTD, and drug exposure (e.g. amiodarone, methotrexate) has been associated with worse clinical outcomes [29]. In this study by LEE et al. [29], unadjusted transplant-free survival was significantly worse in patients who had ILD with a history of inhalational exposure compared to those without. However, this difference did not remain significant after adjustment for gender, age, lung function indices and pack-years of smoking, which might suggest that in addition to these prognostic indices, smoking may independently influence outcomes in the population of exposure-related ILDs. Notably, in that investigation, patients with autoimmune-related ILD subtypes such as IPAF and CTD-ILD also had a high prevalence of inhalational exposures, further supporting the link between autoimmunity and environmental exposures [29]. It has been shown in a cohort of 120 patients with HP that the presence of clinical and serological autoimmunity may portend a poorer prognosis [30]. Another study of 71 patients with coal worker's pneumoconiosis concluded that high-titre rheumatoid factor in these patients may be associated with more severe disease, extra-articular features and rheumatoid nodules [31].

Conversely, autoantibodies have been associated with better clinical outcomes in patients with idiopathic interstitial pneumonia. One study demonstrated that in patients with AIF-ILD a higher ANA titre conferred a better prognosis [5]. Another study examining autoantibodies in patients with IPF found no significant difference between the number of IPF patients and healthy controls in the number of circulating autoantibodies. The presence of autoantibodies was associated with a longer transplant-free survival in IPF patients, although the significance depended on the statistical model used [32].

Our study has several limitations, including the retrospective nature of the study. There is no established time course between the exposure, ANA serology and ILD diagnosis. This, as well as the small sample size and observational design, do not allow for conclusions about causality, although they do allow for an association. There is a selection bias in that only patients with serological testing were included. Although smoking status was noted in most medical records, not all charts documented the presence or absence of other exposures. Because ELISA testing has lower specificity than IFA testing, it may have more false positive tests.

\section{Conclusion}

This study shows that ANA seropositivity is associated with inhalational exposure to environmental antigens in patients with ILD. Our findings suggest that ANA, and by extension, autoimmunity, probably plays a significant role in the pathophysiology of ILD. While we cannot demonstrate causality in this retrospective analysis, these associations provide additional insights into the underlying mechanisms of disease and may offer guidance for future testing and therapies.

Acknowledgements: Our profound appreciation goes to the support staff of the University of Chicago Respiratory Clinical Research Unit, the Interstitial Lung Disease Program (Nancy Trojan, Catherine Brown and Spring Maleckar) and NorthShore University HealthSystem (Evanston, IL, USA) (Daniel Chertok, Mohammad Imran Beig, Paul Chung and Naomi Benlsrael Olive) for their assistance with this study. We also extend our gratitude to the patients with interstitial lung disease who made these research endeavours possible.

Provenance: Submitted article, peer reviewed.

Author contributions: K. Biblowitz, S.K. Bellam and A. Adegunsoye conceived and designed the study; K. Biblowitz, C. Lee, R. Vij, S.K. Bellam and A. Adegunsoye acquired the data for the work; K. Biblowitz, C. Lee, I. Noth, R. Vij, M. 
E. Strek, S.K. Bellam and A. Adegunsoye analysed and interpreted the data; K. Biblowitz, C. Lee, I. Noth, R. Vij, M.E. Strek, S.K. Bellam and A. Adegunsoye drafted the manuscript for important intellectual content; and all authors critically revised the manuscript for important intellectual content, gave final approval of the submitted manuscript and are accountable for all aspects of the work.

Conflict of interest: K. Biblowitz has nothing to disclose. C. Lee has nothing to disclose. D. Zhu has nothing to disclose. I. Noth has nothing to disclose. R. Vij has nothing to disclose. M.E. Strek has received institutional support to conduct interstitial lung disease clinical trials for Boehringer Ingelheim and Galapagos, fees for clinical trial adjudication committee service from Fibrogen, and editorial support from Boehringer Ingelheim. S.K. Bellam has received speaking and advisory board fees from Genentech. A. Adegunsoye has received speaking and advisory board fees from Genentech and Boehringer Ingelheim, and is supported by a career development award from the National Heart, Lung, and Blood Institute (NHLBI K23HL146942).

Support statement: This study was supported by US National Institutes of Health grant K23HL146942.

References

$1 \quad$ Lederer DJ, Martinez FJ. Idiopathic pulmonary fibrosis. N Engl J Med 2018; 378: 1811-1823.

2 GBD 2013 Mortality and Causes of Death Collaborators. Global, regional, and national age-sex specific all-cause and cause-specific mortality for 240 causes of death, 1990-2013: a systematic analysis for the Global Burden of Disease Study 2013. Lancet 2015; 385: 117-171.

3 Kim EJ, Collard HR, King TE. Rheumatoid arthritis associated interstitial lung disease: the relevance of histopathologic and radiographic pattern. Chest 2009; 136: 1397-1405.

4 Mathai SC, Danoff SK. Management of interstitial lung disease associated with connective tissue disease. BMJ 2016; 352: h6819.

5 Vij R, Noth I, Strek ME. Autoimmune-featured interstitial lung disease: a distinct entity. Chest 2011; 140: 1292-1299.

6 Fischer A, Antoniou KM, Brown KK, et al. An official European Respiratory Society/American Thoracic Society research statement: interstitial pneumonia with autoimmune features. Eur Respir J 2015; 46: 976-987.

7 Ryu JH, Colby TV, Hartman TE, et al. Smoking-related interstitial lung diseases: a concise review. Eur Respir J 2001; 17: 122-132.

8 Garcha PS, Gupta S, Kummerfeldt CE. Diffuse parenchymal lung disease. In: Tao L, Khosa S, Pasnick S, et al., eds. The ATS Pulmonary Board Review. 2015, New York, American Thoracic Society; pp. 221-304. Available from: www.thoracic.org/professionals/education/review-for-the-pulmonary-boards.php

9 Turner MT, Samuel SR, Silverstone EJ, et al. Silica exposure and connective tissue disease: an underrecognized association in three Australian artificial stone workers. Am J Respir Crit Care Med 2020; 201: 378-380.

10 Rocha LF, Luppino Assad AP, Marangoni RG, et al. Systemic sclerosis and silica exposure: a rare association in a large Brazilian cohort. Rheumatol Int 2016; 36: 697-702.

11 Ilar A, Alfredsson L, Wiebert P, et al. Occupation and risk of developing rheumatoid arthritis: results from a population-based case-control study. Arthritis Care Res 2018; 70: 499-509.

12 Adegunsoye A, Oldham JM, Bellam SK, et al. African-American race and mortality in interstitial lung disease: a multicentre propensity-matched analysis. Eur Respir J 2018; 51: 1800255.

13 Tan EM, Feltkamp TE, Smolen JS, et al. Range of antinuclear antibodies in "healthy" individuals. Arthritis Rheum 1997; 40: 1601-1611.

14 Kavanaugh A, Tomar R, Reveille J, et al. Guidelines for clinical use of the antinuclear antibody test and tests for specific autoantibodies to nuclear antigens. Arch Pathol Lab Med 2000; 124: 71-81.

15 Copple SS, Sawitzke AD, Wilson AM, et al. Enzyme-linked immunosorbent assay screening then indirect immunofluorescence confirmation of antinuclear antibodies: a statistical analysis. Am J Clin Pathol 2011; 135: 678-684.

16 Prapinjumrune C, Prucktrakul C, Sooktonglarng T, et al. Serum antinuclear antibody in adult Thais. Gerodontology 2017; 34: 86-89.

17 Selmi C, Ceribelli A, Generali E, et al. Serum antinuclear and extractable nuclear antigen antibody prevalence and associated morbidity and mortality in the general population over 15 years. Autoimmun Rev 2016; 15: $162-166$

18 Gniewek RA, Stites DP, McHugh TM, et al. Comparison of antinuclear antibody testing methods: immunofluorescence assay versus enzyme immunoassay. Clin Diagn Lab Immunol 1997; 4: 185-188.

19 Duchemann B, Annesi-Maesano I, Jacobe de Naurois C, et al. Prevalence and incidence of interstitial lung diseases in a multi-ethnic county of Greater Paris. Eur Respir J 2017; 50: 1602419.

20 Bernatsky S, Fournier M, Pineau CA, et al. Associations between ambient fine particulate levels and disease activity in patients with systemic lupus erythematosus (SLE). Environ Health Perspect 2011; 119: 45-49. 
21 American Lung Association. Disparities in the Impact of Air Pollution. www.lung.org/clean-air/outdoors/ who-is-at-risk/disparities/ Date last accessed: 15 June 2021. Date last updated: 20 April 2020.

22 Adegunsoye A, Oldham JM, Husain AN, et al. Autoimmune hypothyroidism as a predictor of mortality in chronic hypersensitivity pneumonitis. Front Med 2017; 4: 170.

23 Pfau JC, Sentissi JJ, Weller G, et al. Assessment of autoimmune responses associated with asbestos exposure in Libby, Montana, USA. Environ Health Perspect 2005; 113: 25-30.

24 Rocha MC, Santos LM, Bagatin E, et al. Genetic polymorphisms and surface expression of CTLA-4 and PD-1 on T cells of silica-exposed workers. Int J Hyg Environ Health 2012; 215: 562-569.

25 Sugiyama D, Nishimura K, Tamaki K, et al. Impact of smoking as a risk factor for developing rheumatoid arthritis: a meta-analysis of observational studies. Ann Rheum Dis 2010; 69: 70-81.

26 Di Giuseppe D, Discacciati A, Orsini N, et al. Cigarette smoking and risk of rheumatoid arthritis: a dose-response meta-analysis. Arthritis Res Ther 2014; 16: R61.

27 Chang K, Yang SM, Kim SH, et al. Smoking and rheumatoid arthritis. Int J Mol Sci 2014; 15: 22279-22295.

28 Rowley B, Monestier M. Mechanisms of heavy metal-induced autoimmunity. Mol Immunol 2005; 42: 833-888.

29 Lee $\mathrm{CT}$, Adegunsoye A, Chung JH, et al. Characteristics and prevalence of domestic and occupational inhalational exposures across interstitial lung diseases. Chest 2021; 160: 209-218.

30 Adegunsoye A, Oldham JM, Demchuk C, et al. Predictors of survival in coexistent hypersensitivity pneumonitis with autoimmune features. Respir Med 2016; 114: 53-60.

31 Kart L, Sarikaya S, Gurel A, et al. Rheumatoid factor seropositivity and rheumatoid symptoms in coal worker's pneumoconiosis. Clin Rheumatol 2003; 22: 365-366.

32 Lee JS, Kim EJ, Lynch KL, et al. Prevalence and clinical significance of circulating autoantibodies in idiopathic pulmonary fibrosis. Respir Med 2013; 107: 249-255. 